PROCEEDINGS OF THE

AMERICAN MATHEMATICAL SOCIETY

Volume 137, Number 5, May 2009, Pages 1649-1656

S 0002-9939(08)09735-9

Article electronically published on December 10, 2008

\title{
FIRST NEIGHBORHOOD COMPLETE IDEALS IN TWO-DIMENSIONAL MUHLY LOCAL DOMAINS ARE PROJECTIVELY FULL
}

\author{
RAYMOND DEBREMAEKER
}

(Communicated by Bernd Ulrich)

\begin{abstract}
Let $(R, \mathcal{M})$ be a two-dimensional Muhly local domain, i.e., an integrally closed Noetherian local domain with algebraically closed residue field and the associated graded ring an integrally closed domain.

Motivated by recent work of Ciuperca, Heinzer, Ratliff and Rush on projectively full ideals, we prove that every complete ideal adjacent to the maximal ideal $\mathcal{M}$ is projectively full.
\end{abstract}

\section{INTRODUCTION}

This paper is inspired by a result of Ciuperca, Heinzer, Ratliff Jr., and Rush on projectively full ideals. In order to explain this we recall some notions and results from their work.

Two ideals $I$ and $J$ in a commutative Noetherian ring $R$ are called projectively equivalent if there exist positive integers $m$ and $n$ such that $I^{m}$ and $J^{n}$ have the same integral closure, i.e., $\overline{I^{m}}=\overline{J^{n}}$.

The notion of projectively equivalent ideals was introduced by P. Samuel in [14]. This concept was further studied by McAdam, Ratliff Jr., and Sally in 12, where it is proved that if $I$ and $J$ are projectively equivalent, then $I$ and $J$ have the same Rees valuations, i.e., $T(I)=T(J)$, where $T()$ denotes the set of the Rees valuations. Moreover the values of $I$ and $J$ with respect to these Rees valuations are proportional.

In [1] Ciuperca, Heinzer, Ratliff Jr., and Rush have further developed the relationship between projective equivalence of ideals and Rees valuations. In that paper the authors also introduced the notion of projectively full ideal. A regular ideal $I$ in a Noetherian ring $R$ is called projectively full if $\frac{I^{n}}{}$ with $n \in \mathbb{N}_{+}$are the only integrally closed ideals that are projectively equivalent to $I$.

The authors have continued the study of projectively full ideals in 2 .

In the special case where $(R, \mathcal{M})$ is a two-dimensional regular local ring with algebraically closed residue field, the complete ideals $I$ adjacent to $\mathcal{M}$ (i.e., length $\left.\left(\frac{\mathcal{M}}{I}\right)=1\right)$ are projectively full.

Received by the editors May 8, 2008, and, in revised form, August 6, 2008.

2000 Mathematics Subject Classification. Primary 13B22, $13 \mathrm{H} 10$.

Key words and phrases. First neighborhood complete ideal, Muhly local domain, projectively equivalent ideals, projectively full ideal.

(C)2008 American Mathematical Society Reverts to public domain 28 years from publication 
To see this, we first remark that $I$ is simple, i.e., not a product of two proper ideals. Therefore, it follows from Zariski's theory of complete ideals (see [15]) that $I$ has only one Rees valuation, say $w$. Because of [1, Theorem 3.4] it follows that the ideals $J$ projectively equivalent to $I$ are the ideals $J$ such that $T(J)=\{w\}$. With this in mind, we see that it follows from Zariski's Unique Factorization Theorem that the only complete ideals that are projectively equivalent to $I$ are the ideals $\overline{I^{n}}$ with $n \in \mathbb{N}_{+}$, i.e., $I$ is projectively full. One could try to generalize this result to the more general case where $(R, \mathcal{M})$ is a two-dimensional normal local domain with algebraically closed residue field and the associated graded ring an integrally closed domain. These local domains will be called two-dimensional Muhly local domains in the rest of this paper.

Unfortunately the above argument does not work anymore in this case because we will see in the next section that the ideal $I$ has more than one Rees valuation if $R$ is not regular.

But Ciuperca, Heinzer, Ratliff Jr., and Rush in [2, Corollary 3.7] have proved the following nice result: "Let $(R, \mathcal{M})$ be a regular local domain of dimension $\geq 2$ and let $x_{1}, \cdots, x_{d}$ be a regular system of parameters. Then every ideal of the form $I=\left(x_{1}^{n}, x_{2}, \cdots, x_{d}\right) R$ is projectively full."

This result, combined with the fact (see Section 2) that in a two-dimensional Muhly local domain $(R, \mathcal{M})$ a complete ideal $I$ adjacent to $\mathcal{M}$ is of the form

$$
I=\left(x_{1}^{2}, x_{2}, \cdots, x_{n}\right) R
$$

for a suitable minimal ideal basis $x_{1}, \cdots, x_{n}$ of $\mathcal{M}$, inspired us to prove the following result.

Theorem 1. Let $(R, \mathcal{M})$ be a two-dimensional Muhly local domain and I a complete ideal adjacent to $\mathcal{M}$. Then I is projectively full.

For some reason explained in the next section, complete ideals adjacent to $\mathcal{M}$ are called first neighborhood complete ideals of $R$. This combined with the theorem explains the result mentioned in the title of this paper.

Note that in this paper we will use the symbol $\subset$ to mean "contained in or equal to".

\section{Preliminaries on First neighborhood COMPlete ideals}

Let $(R, \mathcal{M})$ be a two-dimensional normal Noetherian local domain with algebraically closed residue field and suppose that the associated graded ring is an integrally closed domain. It then follows that the $\mathcal{M}$-adic order function $v_{\mathcal{M}}$ is a valuation of the quotient field $K$ of $R$ and $\mathcal{M}^{n}$ is an integrally closed ideal for all $n \in \mathbb{N}$. Moreover, it follows that the blowup $B l_{\mathcal{M}}(R)$ of $R$ at $\mathcal{M}$ is a desingularization of $R$. (The associated graded ring $\operatorname{gr}_{\mathcal{M}}(R)$ is an integrally closed domain, so the closed fibre of the blowup of $R$ at $\mathcal{M}$ is a non-singular curve, and this implies that every local ring of $B l_{\mathcal{M}}(R)$ is regular [8, p. 403], [10, p. 259].)

These local domains (supplemented with one more condition) have been studied by H.T. Muhly [13, thereby generalizing some aspects of Zariski's theory of complete ideals in two-dimensional regular local rings. Therefore these local domains will be called two-dimensional Muhly local domains throughout this paper.

Parts of the theory of Zariski have been extended to other classes of normal local rings by several other authors, including Cutkosky [3, 4], Fernández-Sánchez [6, 7], Göhner [8, Huneke and Sally [9] and Lipman [10, 11]. 
An ideal $I$ is said to be integrally closed or complete if $\bar{I}=I$, where $\bar{I}$ denotes the integral closure of $I$; i.e., $\bar{I}=\{r \in R \mid r$ satisfies an equation of the form $r^{n}+i_{1} r^{n-1}+\cdots+i_{n}=0$ with $i_{k} \in I^{k}$ for $\left.k=1, \cdots, n\right\}$. In case $\overline{I^{n}}=I^{n}$ for all $n \in \mathbb{N}_{+}, I$ is called a normal ideal.

In this note we are concerned with complete ideals $I$ adjacent to $\mathcal{M}$, i.e., length $\left(\frac{\mathcal{M}}{I}\right)=1$, in two-dimensional Muhly local domains $(R, \mathcal{M})$. Since complete ideals adjacent to $\mathcal{M}$ are well understood in the two-dimensional regular case, $(R, \mathcal{M})$ will from now on denote a two-dimensional Muhly local domain that is not regular.

We now discuss a few results about complete ideals adjacent to $\mathcal{M}$ in a twodimensional Muhly local domain $(R, \mathcal{M})$. A more complete treatment of this matter can be found in the forthcoming paper [5]. First we will make clear how to produce complete ideals adjacent to $\mathcal{M}$, namely by taking the inverse transform of the maximal ideal of the immediate quadratic transforms of $(R, \mathcal{M})$ (terminology is explained below).

Let $\left(R^{\prime}, \mathcal{M}^{\prime}\right)$ be an immediate (or first) quadratic transform of $(R, \mathcal{M})$, that is

$$
R^{\prime}=R\left[\frac{\mathcal{M}}{x_{1}}\right]_{M_{1}}
$$

where $x_{1}, x_{2}, \cdots, x_{n}$ is a minimal ideal basis of $\mathcal{M}$ and $M_{1}$ is a maximal ideal in $R\left[\frac{\mathcal{M}}{x_{1}}\right]$ lying over $\mathcal{M}$.

In section 3 of [5] it is shown that a minimal ideal basis $x_{1}, x_{2}, \cdots, x_{n}$ of $\mathcal{M}$ can be chosen such that

$$
M_{1}=\left(x_{1}, \frac{x_{2}}{x_{1}}, \cdots, \frac{x_{n}}{x_{1}}\right) R\left[\frac{\mathcal{M}}{x_{1}}\right]
$$

because the residue field $\frac{R}{\mathcal{M}}$ is algebraically closed.

Now let us consider the inverse transform $I$ of $\mathcal{M}^{\prime}$ in $R$, i.e.,

$$
I:=x_{1} \mathcal{M}^{\prime} \cap R \text {. }
$$

A number of properties of the ideal $I$ are collected in the following lemma.

Lemma 1. With the same conventions as above we have

(i) $I=\left(x_{1}^{2}, x_{2}, \cdots, x_{n}\right) R$.

(ii) $I$ is a simple complete $\mathcal{M}$-primary ideal and $I \subset \mathcal{M}$ are adjacent.

(iii) $\left(R^{\prime}, \mathcal{M}^{\prime}\right)$ is the unique immediate base point of $I$; that is, $\left(R^{\prime}, \mathcal{M}^{\prime}\right)$ is the unique two-dimensional local ring of $B \ell_{\mathcal{M}}(R)$ such that $I R^{\prime}$ is not a principal ideal.

(iv) $\mathcal{M}^{\prime}$ is the transform of $I$ in $R^{\prime}$, i.e., $I R^{\prime}=x_{1} \mathcal{M}^{\prime}$.

(v) $T(I)=\left\{v_{\mathcal{M}}, w\right\}$ where $w$ denotes the ord $_{R^{\prime} \text {-valuation. }}$

Proof. (i) One has the following inclusions:

$$
\left(x_{1}^{2}, x_{2}, \cdots, x_{n}\right) R \subset x_{1} M_{1} \cap R \subset \mathcal{M} .
$$

Since $\left(x_{1}^{2}, x_{2}, \cdots, x_{n}\right) R \subset \mathcal{M}$ are adjacent and $x_{1} M_{1} \cap R=\mathcal{M}$ is impossible, it follows that

$$
I=x_{1} M_{1} \cap R=\left(x_{1}^{2}, x_{2}, \cdots, x_{n}\right) R .
$$

(ii) and (iv) follow immediately from (i).

(iii) Since $\operatorname{IR}\left[\frac{\mathcal{M}}{x_{1}}\right]=x_{1} M_{1}$, it follows that $\left(R^{\prime}, \mathcal{M}^{\prime}\right)$ is the only immediate base point of $I$ on the chart $R\left[\frac{\mathcal{M}}{x_{1}}\right]$. If $i \in\{2, \cdots, n\}$, then we have that $\operatorname{IR}\left[\frac{\mathcal{M}}{x_{i}}\right]=$ $x_{i} R\left[\frac{\mathcal{M}}{x_{i}}\right]$, so there are no immediate base points of $I$ on $R\left[\frac{\mathcal{M}}{x_{i}}\right]$. This shows that $\left(R^{\prime}, \mathcal{M}^{\prime}\right)$ is the unique immediate base point of $I$. 
(v) We know that $\left(R^{\prime}, \mathcal{M}^{\prime}\right)$ is the only immediate base point of $I$ and $\mathcal{M}^{\prime}$ is its transform in $R^{\prime}$. This implies that $T(I \mathcal{M})=\left\{v_{\mathcal{M}}, w\right\}$; hence

$$
T(I) \subset\left\{v_{\mathcal{M}}, w\right\} .
$$

Since the two-dimensional Muhly local domain $(R, \mathcal{M})$ is not regular, we have $v_{\mathcal{M}} \in T(I)$ because of Theorem 3.3 in [5. Finally, $w \in T(I)$ since otherwise $I$ would be a simple complete $\mathcal{M}$-primary ideal with $T(I)=\left\{v_{\mathcal{M}}\right\}$, implying that $I=\mathcal{M}$, a contradiction.

Next we prove that the complete $\mathcal{M}$-primary ideals adjacent to $\mathcal{M}$ obtained by considering the inverse transform in $R$ of the maximal ideal of the immediate transforms of $R$ are in fact all the complete $\mathcal{M}$-primary ideals adjacent to $\mathcal{M}$. More precisely we have the following result.

Lemma 2. The mapping that associates to the maximal ideal $\mathcal{M}^{\prime}$ of an immediate quadratic transform $R^{\prime}$ of $R$ its inverse transform $I$ in $R$ is a one-to-one correspondence between the set of the immediate quadratic transforms of $(R, \mathcal{M})$ and the set of the complete $\mathcal{M}$-primary ideals adjacent to $\mathcal{M}$. The inverse mapping consists in associating to I its unique immediate base point.

Proof. The existence of the mapping follows immediately from Lemma 1. Next we prove the surjectivity of this mapping. So let $I$ be any complete $\mathcal{M}$-primary ideal adjacent to $\mathcal{M}$. Then $I$ has at least one immediate base point, since otherwise one would have that $T(I) \subset T(\mathcal{M})=\left\{v_{\mathcal{M}}\right\}$ and thus $I=\mathcal{M}$, which is impossible.

Let $\left(R^{\prime}, \mathcal{M}^{\prime}\right)$ denote an immediate base point of $I$. We claim that $I$ is the inverse transform of $\mathcal{M}^{\prime}$ in $R$. To see this, let us suppose that a minimal ideal basis $x_{1}, x_{2}, \cdots, x_{n}$ of $\mathcal{M}$ has been chosen as explained just before Lemma 1 , so $R^{\prime}=R\left[\frac{\mathcal{M}}{x_{1}}\right]_{M_{1}}$ with $M_{1}=\left(x_{1}, \frac{x_{2}}{x_{1}}, \cdots, \frac{x_{n}}{x_{1}}\right)$.

In $R^{\prime}$ we have

$$
I R^{\prime}=x_{1} I^{\prime}
$$

with $I^{\prime}$ an ideal of $R^{\prime}$ such that $I^{\prime} \neq R^{\prime}$ because $R^{\prime}$ is a base point of $I$.

In $R$ we have the following chain of inclusions:

$$
I \subset x_{1} I^{\prime} \cap R \subset x_{1} \mathcal{M}^{\prime} \cap R \subset \mathcal{M} .
$$

Since $I \subset \mathcal{M}$ are adjacent, it follows that

$$
I=x_{1} \mathcal{M}^{\prime} \cap R,
$$

i.e., that $I$ is the inverse transform of $\mathcal{M}^{\prime}$ in $R$. Note that it follows from Lemma 1 that $I$ has the following form:

$$
I=\left(x_{1}^{2}, x_{2}, \cdots, x_{n}\right) R,
$$

which implies that $\left(R^{\prime}, \mathcal{M}^{\prime}\right)$ is in fact the only immediate base point of $I$.

Remark 1. Because of the previous result, complete ideals adjacent to $\mathcal{M}$ will be called first neighborhood complete ideals of the two-dimensional Muhly local domain $(R, \mathcal{M})$. 


\section{Proof of the MAIN THEOREM}

We are now ready to prove the theorem announced in the introduction.

Let $(R, \mathcal{M})$ be a two-dimensional Muhly local domain (not regular) and let $I$ be any first neighborhood complete ideal of $R$. As we have seen in the previous section, the ideal $I$ has a unique immediate base point, say $\left(R^{\prime}, \mathcal{M}^{\prime}\right)$, and a minimal ideal basis $x_{1}, \cdots, x_{n}$ of $\mathcal{M}$ can be chosen such that

$$
R^{\prime}=R\left[\frac{\mathcal{M}}{x_{1}}\right]_{M_{1}} \text { with } M_{1}:=\left(x_{1}, \frac{x_{2}}{x_{1}}, \cdots, \frac{x_{n}}{x_{1}}\right) R\left[\frac{\mathcal{M}}{x_{1}}\right] .
$$

It follows that

$$
I=\left(x_{1}^{2}, x_{1}, \cdots, x_{n}\right) R
$$

and

$$
\operatorname{IR}\left[\frac{\mathcal{M}}{x_{1}}\right]=x_{1} M_{1}
$$

Further, this implies that $T(I)=\left\{v_{\mathcal{M}}, w\right\}$, where $w$ denotes the $\operatorname{ord}_{R^{\prime}}$-valuation.

In order to prove that $I$ is projectively full, we have to show that any complete $\mathcal{M}$-primary ideal $J$ projectively equivalent to $I$ is of the following form:

$$
\overline{I^{\nu}} \text { with } \nu \in \mathbb{N}_{+} \text {. }
$$

Since $J$ is projectively equivalent to $I$ we have that

$$
T(J)=T(I)=\left\{v_{\mathcal{M}}, w\right\},
$$

implying that $J$, just like $I$, has $\left(R^{\prime}, \mathcal{M}^{\prime}\right)$ as its unique immediate base point.

Let $r:=\operatorname{ord}_{R}(J)$. Then we have in $R\left[\frac{\mathcal{M}}{x_{1}}\right]$,

$$
J R\left[\frac{\mathcal{M}}{x_{1}}\right]=x_{1}^{r} \mathcal{J}
$$

where $\mathcal{J}$ denotes an ideal of $R\left[\frac{\mathcal{M}}{x_{1}}\right]$ not contained in any ht 1-prime. Since $R^{\prime}=$ $R\left[\frac{\mathcal{M}}{x_{1}}\right]_{M_{1}}$ is the unique immediate base point of $J$, it follows that $M_{1}$ is the unique prime ideal of $R\left[\frac{\mathcal{M}}{x_{1}}\right]$ containing $\mathcal{J}$. Thus $\mathcal{J}$ is an $M_{1}$-primary ideal of $R\left[\frac{\mathcal{M}}{x_{1}}\right]$, which is called the transform of $J$ in $R\left[\frac{\mathcal{M}}{x_{1}}\right]$. Note that we do not know whether $\mathcal{J}$ is complete or not.

Localizing at $M_{1}$ yields

$$
J R^{\prime}=x_{1}^{r} \mathcal{J}_{M_{1}},
$$

which means that the transform $J^{\prime}$ of $J$ in $R^{\prime}$ is given by

$$
J^{\prime}=\mathcal{J}_{M_{1}}
$$

From $J R^{\prime}=x_{1}^{r} J^{\prime}$ it follows that

$$
\overline{J R}^{\prime}=x_{1}^{r} \overline{J^{\prime}} .
$$

We claim: the complete $\mathcal{M}^{\prime}$-primary ideal $\overline{J^{\prime}}$ of $R^{\prime}$ has only one Rees valuation, namely $w$, i.e.,

$$
T\left(\overline{J^{\prime}}\right)=\{w\}
$$

To prove this claim, let us suppose that $w^{\prime}$ is a Rees valuation of $\overline{J^{\prime}}$ with $w^{\prime} \neq w$.

We now show this leads to a contradiction. Since $w^{\prime}$ is a Rees valuation of $J^{\prime}$, we have that

$$
\mathcal{W}^{\prime} \in \overline{B l_{J^{\prime}}\left(R^{\prime}\right)}
$$


where $\mathcal{W}^{\prime}$ denotes the valuation ring of $w^{\prime}$. Because $J R^{\prime}=x_{1}^{r} J^{\prime}$ we have

$$
\overline{B l_{J^{\prime}}\left(R^{\prime}\right)}=\overline{B l_{J R^{\prime}}\left(R^{\prime}\right)} .
$$

From the fact that $\left(R^{\prime}, \mathcal{M}^{\prime}\right)$ is the only immediate base point of $J$ it follows that the blowup $B l_{J \mathcal{M}}(R)$ is obtained by blowing up $R$ at $\mathcal{M}$ and then blowing up the local ring $\left(R^{\prime}, \mathcal{M}^{\prime}\right) \in B l_{\mathcal{M}}(R)$ at $J R^{\prime}$. This implies that

$$
\mathcal{W}^{\prime} \in \overline{B l_{J \mathcal{M}}(R)}
$$

and it follows that

$$
w^{\prime} \in T(J \mathcal{M})=T(\mathcal{M}) \cup T(J),
$$

where $T(\mathcal{M})=\left\{v_{\mathcal{M}}\right\}$ and $T(J)=\left\{v_{\mathcal{M}}, w\right\}$. As $w^{\prime} \neq w$, we have that $w^{\prime}=v_{\mathcal{M}}$, which implies that $\mathcal{W}^{\prime} \in B l_{\mathcal{M}}(R)$. Since $\mathcal{W}^{\prime}$ dominates the local ring $R^{\prime} \in B l_{\mathcal{M}}(R)$, we have a contradiction. So $\overline{J^{\prime}}$ cannot have Rees valuations $\neq w$, and this proves our claim.

Since the complete $\mathcal{M}^{\prime}$-primary ideal $\overline{J^{\prime}}$ in the two-dimensional regular local ring $\left(R^{\prime}, \mathcal{M}^{\prime}\right)$ has only one Rees valuation $w$, it follows from Zariski's Unique Factorization Theorem [15] that $\overline{J^{\prime}}$ is a power of the simple complete $\mathcal{M}^{\prime}$-primary ideal having $w$ as its unique Rees valuation. Thus

$$
\overline{J^{\prime}}=\mathcal{M}^{\prime \nu} \text { with } \nu \in \mathbb{N}_{+} .
$$

This together with $\overline{J R^{\prime}}=x_{1}^{r} \overline{J^{\prime}}$ yields

$$
\overline{J R^{\prime}}=x_{1}^{r} \mathcal{M}^{\prime \nu}=x_{1}^{r}\left(M_{1}^{\nu}\right)_{M_{1}} .
$$

Since $J^{\prime}=\mathcal{J}_{M_{1}}$, we also have

$$
\overline{J R^{\prime}}=x_{1}^{r} \overline{\left(\mathcal{J}_{M_{1}}\right)} .
$$

From (1) and (2) it follows that

$$
x_{1}^{r} \overline{\left(\mathcal{J}_{M_{1}}\right)}=x_{1}^{r}(\overline{\mathcal{J}})_{M_{1}}=x_{1}^{r}\left(M_{1}^{\nu}\right)_{M_{1}} .
$$

So

$$
(\overline{\mathcal{J}})_{M_{1}}=\left(M_{1}^{\nu}\right)_{M_{1}} \text {, whence } \overline{\mathcal{J}}=M_{1}^{\nu} \text {. }
$$

Thus we have

$$
\overline{J R\left[\frac{\mathcal{M}}{x_{1}}\right]}=x_{1}^{r} \overline{\mathcal{J}}=x_{1}^{r} M_{1}^{\nu} .
$$

Now we claim that $r=\nu$. This can be seen as follows. As $J$ is projectively equivalent to $I$, we have a relation of the form

$$
\overline{I^{i}}=\overline{J^{j}} \quad \text { with } i, j \in \mathbb{N}_{+} .
$$

Taking the $\operatorname{ord}_{R^{-}}$value of both sides, we obtain

$$
i=r j
$$

and hence

$$
\overline{J^{j}}=\overline{I^{r j}} .
$$

It follows that

$$
\overline{J^{j} R^{\prime}}=\overline{I^{r j} R^{\prime}} .
$$

Now $I R\left[\frac{\mathcal{M}}{x_{1}}\right]=x_{1} M_{1}$ implies that $I R^{\prime}=x_{1} \mathcal{M}^{\prime}$ and thus $I^{r j} R^{\prime}=x_{1}^{r j} \mathcal{M}^{\prime r j}$. This implies that

$$
\overline{J^{j} R^{\prime}}=\overline{x_{1}^{r j} \mathcal{M}^{\prime r j}}=x_{1}^{r j} \mathcal{M}^{\prime r j}
$$


On the other hand, from (1) we have

$$
\overline{J R^{\prime}}=x_{1}^{r} \mathcal{M}^{\prime \nu}
$$

and thus

$$
\overline{J^{j} R^{\prime}}=\left(\overline{J R^{\prime}}\right)^{j}=x_{1}^{r j} \mathcal{M}^{\prime \nu j} .
$$

Comparing (3) and (4) gives

$$
\mathcal{M}^{\prime \nu j}=\mathcal{M}^{\prime r j},
$$

which implies that $\nu=r$.

Putting $\nu=r$ in $(*)$ yields

$$
\overline{J R\left[\frac{\mathcal{M}}{x_{1}}\right]}=x_{1}^{r} M_{1}^{r} .
$$

Now we have the following chain of inclusions:

$$
J \subset J R\left[\frac{\mathcal{M}}{x_{1}}\right] \cap R \subset \overline{J R\left[\frac{\mathcal{M}}{x_{1}}\right]} \cap R \subset J \mathcal{V} \cap J \mathcal{W} \cap R,
$$

where $\mathcal{V}$ denotes the valuation ring of $v_{\mathcal{M}}=\operatorname{ord}_{R}$ and $\mathcal{W}$ the valuation ring of $w$.

Since $T(J)=\left\{v_{\mathcal{M}}, w\right\}$ and $J$ is complete, we have

$$
J \mathcal{V} \cap J \mathcal{W} \cap R=J ;
$$

hence

$$
J=\overline{J R\left[\frac{\mathcal{M}}{x_{1}}\right]} \cap R=x_{1}^{r} M_{1}^{r} \cap R .
$$

Finally one can verify that

$$
x_{1}^{r} M_{1}^{r} \cap R=\overline{I^{r}},
$$

which shows that $J=\overline{I^{r}}$, i.e., that $I$ is projectively full.

\section{REFERENCES}

[1] C. Ciuperca, W.J. Heinzer, L.J. Ratliff Jr., D.E. Rush, Projectively equivalent ideals and Rees valuations, J. Algebra 282 (2004) 140-156. MR2095576 (2005g:13014)

[2] C. Ciuperca, W.J. Heinzer, L.J. Ratliff Jr., D.E. Rush, Projectively full ideals in Noetherian rings, J. Algebra 304 (1) (2006) 73-93. MR2255821 (2007m:13009)

[3] S.D. Cutkosky, On unique and almost unique factorization of complete ideals, Amer. J. Math. 111 (3) (1989) 417-433. MR1002007 (90j:14016a)

[4] S.D. Cutkosky, On unique and almost unique factorization of complete ideals. II, Invent. Math. 98 (1) (1989) 59-74. MR.1010155 (90j:14016b)

[5] R. Debremaeker, First neighborhood complete ideals in two-dimensional Muhly local domains, J. Pure Appl. Algebra, to appear.

[6] J. Fernández-Sánchez, On sandwiched singularities and complete ideals, J. Pure Appl. Algebra 185 (1-3) (2003) 165-175. MR2006424 (2005a:14008)

[7] J. Fernández-Sánchez, Factorization of complete ideals in normal birational extensions in dimension two, J. Algebra 314 (2007) 344-361. MR2331766

[8] H. Göhner, Semifactoriality and Muhly's condition $\mathrm{N}$ in two dimensional local rings, J. Algebra 34 (1975) 403-429. MR0379489 (52:394)

[9] C. Huneke, J.D. Sally, Birational extensions in dimension two and integrally closed ideals, J. Algebra 115 (2) (1988) 481-500. MR943272 (89e:13025)

[10] J. Lipman, Rational singularities, with applications to algebraic surfaces and unique factorization, Inst. Hautes Études Sci. Publ. Math. 36 (1969) 195-279. MR0276239 (43:1986)

[11] J. Lipman, On complete ideals in regular local rings, in: Algebraic Geometry and Commutative Algebra (in honor of M. Nagata), vol. I, Kinokuniya, Tokyo, 1988, pp. 203-231. MR977761 (90g:14003) 
[12] S. McAdam, L.J. Ratliff Jr., J.D. Sally, Integrally closed projectively equivalent ideals, in: Commutative Algebra, Math. Sci. Res. Inst. Publ., vol. 15, Springer, New York, 1989, pp. 391405. MR:1015530 (91b:13007)

[13] H.T. Muhly, On the existence of asymptotically irreducible ideals, J. London Math. Soc. 40 (1965) 99-107. MR0170904 (30:1139)

[14] P. Samuel, Some asymptotic properties of powers of ideals, Ann. of Math. (2) 56 (1952) 11-21. MR0049166 (14:128c)

[15] O. Zariski, P. Samuel, Commutative Algebra, vol. II, Van Nostrand, New York, 1960. MR0120249 (22:11006)

Department of Mathematics, Katholieke Universiteit, Leuven, Celestijnenlaan 200B-Box 2400, BE-3001 Leuven, Belgium

E-mail address: raymond.debremaeker@wis.kuleuven.be 